\title{
Status of programmed death-ligand 1 expression in sarcomas
}

\author{
Hyung Kyu Park ${ }^{1 \dagger}$, Mingi Kim ${ }^{2,3+}$, Minjung Sung ${ }^{3}$, Seung Eun Lee ${ }^{1}$, Yu Jin Kim ${ }^{3 *}$ and Yoon-La Choi ${ }^{2,3,4^{*}}$
}

\begin{abstract}
Background: Sarcomas are challenging to study because of their rarity and histomorphological complexity. PD1 and PD-L1 inhibitors showed a promising anti-tumor effect in solid tumors, where a relationship between PD-L1 expression and the objective response has been evidenced.

Methods: In this study, we examined PD-L1 expression in 16 bone and soft tissue sarcoma cell lines of 11 different subtypes by means of western blot, flow cytometry and immunocytochemistry, and in 230 FFPE patient-derived tumor tissues by means of immunohistochemistry using three different antibody clones. The association between PD-L1 expression and clinicopathological features was evaluated.
\end{abstract}

Results: We demonstrated that PD-L1 protein is highly expressed in pleomorphic rhabdomyosarcoma, fibrosarcoma, and dedifferentiated liposarcoma (DDLPS) cell lines. From the tissue microarray, undifferentiated pleomorphic sarcoma showed $\geq 1 \%$ immunoreactivity in 20\%, 17.6\%, and 16.3\% of the cases with PD-L1 22C3, SP263, and SP142 antibodies, respectively. In whole sections stained with a PD-L1 22C3 antibody, DDLPS showed $\geq 1 \%$ immunoreactivity in $21.9 \%$ of the cases. In DDLPS group, cases with $\geq 1 \%$ PD-L1 expression showed statistically significantly worse recurrence-free survival $(P=0.027)$ and overall survival $(P=0.017)$ rates. Upon interferon-gamma treatment, the mRNA expression levels of PD-L1 were elevated in the HS-RMS-1, LIPO-224B, MLS1765, RH30, and RH41 cell lines.

Conclusions: We found that the expression of PD-L1 in sarcoma differs depending on the histologic subtype and the PD-L1 antibody clones. These results may serve as primary data for the selection of appropriate patients when applying PD1/PD-L1 inhibitor therapy in sarcoma.

Keywords: Sarcoma, PD-L1, DDLPS, UPS, IFN-

\section{Background}

Sarcomas are malignant mesenchymal tumors that account for approximately $1 \%$ of adult solid cancers [1]. Sarcomas can be divided into more than 50 distinct histological subtypes, and many of these subtypes are not limited to a specific location of the body [2]. Due to its rarity and morphological variability, the clinical and pathological study of sarcomas have been limited, and

\footnotetext{
*Correspondence: bubble@skku.edu; ylachoi@skku.edu

${ }^{\dagger}$ Hyung Kyu Park and Mingi Kim contributed equally to this work

${ }^{3}$ Laboratory of Cancer Genomics and Molecular Pathology, Samsung

Medical Center, Sungkyunkwan University School of Medicine, Irwon-ro

81, Gangnam-gu, Seoul 06351, South Korea

${ }^{4}$ Department of Pathology and Translational Genomics, Samsung

Medical Center, Sungkyunkwan University School of Medicine, Irwon-ro

81, Gangnam-gu, Seoul 06351, South Korea

Full list of author information is available at the end of the article
}

the mainstay of sarcoma treatment has not changed for decades [3, 4]. Surgical resection with enough safety margins remains as the only curative therapeutic option despite its limited indication and several complications. In cases with inoperable tumors, doxorubicin and ifosfamide have been used for more than 30 years and remain the mainstay for treatments. However, these cytotoxic agents are known to provide overall response rates of only about $25 \%$ in the first-line setting and are currently used for palliative, but not curative, purposes [5]. There is still a need for new treatment methods that surpass previous therapies.

After the discovery of programmed cell death protein 1 (PD-1) in 1992, PD-1 and PD-L1 have been revealed to have a fundamental role in cancer immune surveillance [6, 7]. Anti-PD-1 therapies were approved for 
melanoma, non-small-cell lung cancer, and various solid tumors worldwide [8-10]. Also in cases of sarcoma, several previous studies have reported that more than $50 \%$ of sarcomas, including leiomyosarcoma, dedifferentiated liposarcoma (DDLPS), undifferentiated pleomorphic sarcoma (UPS), osteosarcoma, epithelioid sarcoma, and other sarcomas, showed PD-L1 expression in tumor cells [11-13]. However, recently the SARC028 trial report described that only $4 \%(3 / 70)$ of the sarcoma biopsy samples (all three were from patients with UPS) were immunopositive for PD-L1 in more than $1 \%$ of tumor cells [14]. This study also reported that $11 \%(9 / 80)$ of the patients with sarcomas showed an objective response, especially in patients with undifferentiated sarcomas $(4 / 10)$ and liposarcomas $(2 / 10)$. This result is promising but also resulted in several new questions regarding the PD-L1 immunohistochemical expression rate and its role in practice.

To gain insight into the PD-L1 expression pattern in various patients with sarcomas, we examined the PD-L1 expression using various cell lines and patient tissues including both TMA and whole sections and evaluated the association between PD-L1 expression and clinicopathological features in patients with sarcomas.

\section{Methods}

\section{Patient tissue specimens}

A total of 230 archival formalin-fixed paraffin-embedded (FFPE) soft tissue sarcoma tissue samples, each from a different patient, were collected at the Samsung Medical Center in Seoul, Korea. Ten myxoid liposarcomas, 33 DDLPSs, and 100 UPSs were collected as tissue microarrays (TMAs), while 87 samples were analyzed as whole section from FFPE tissue blocks and comprised 32 DDLPSs, 24 myxoid liposarcomas, and 31 osteosarcomas. This study was approved by the Institutional Review Board of Samsung Medical Center in Seoul, Korea (IRB file No. 2018-03-143). Informed consents were waived by the board.

\section{Cell lines, reagents, and IFN- $\gamma$ treatment}

Human soft tissue sarcoma cell lines were obtained from American Type Culture Collection (ATCC), Korean Cell Line Bank (KCLB), and other laboratories detailed in Additional file 1: Table S1. Each cell line was grown in appropriate culture medium (Additional file 1: Table S2) with $10 \%$ fetal bovine serum (Gibco, 16000-044) and 1\% antibiotic-antimycotic 100× (Gibco, 15240-112). Cell lines were tested and validated for mycoplasma detection and human cell line authentication (STR DNA profiling) using AmpFLSTR ${ }^{\mathrm{TM}}$ Identifiler PCR Amplification Kit (Thermo Fisher Scientific, 4322288). For IFN- $\gamma$ treatment, each cell line was seeded into six-well plates and treated with IFN- $\gamma$ (R\&D systems, 285-IF-100; 50 or $100 \mathrm{ng} / \mathrm{ml}$ ) or BSA (Thermo Fisher Scientific, 23209; 50 or $100 \mathrm{ng} / \mathrm{ml}$ ) as controls and incubated at $37{ }^{\circ} \mathrm{C}$ for $48 \mathrm{~h}$.

\section{Western blot}

Cells were lysed in RIPA buffer $(0.1 \%$ SDS, $0.5 \%$ sodium deoxycholate, $250 \mathrm{Mm} \mathrm{NaCl}, 1 \%$ Triton X-100 and $50 \mathrm{Mm} \mathrm{pH} 8.0$ Tris) containing a phosphatase inhibitor and protease inhibitor cocktail tablets (Roche) and quantified using Pierce ${ }^{\mathrm{TM}}$ BCA Protein Assay kit (Thermo Fisher Scientific, 23227) according to the manufacturer's instructions. One hundred micrograms of total protein from cells were separated by $10 \%$ SDS-PAGE and transferred to nitrocellulose membrane (Pall Corporation), then the membranes were blocked with $5 \%$ nonfat milk in $1 \times$ TBST (Tris-buffered saline with Tween 20). Proteins were probed with the following primary antibodies: monoclonal anti-PD-L1 (Cell Signaling Technology, E1L3N, 1:3000) and anti- $\beta$-actin antibodies (Santa Cruz, sc-47778, 1:1000), and washed three times with $1 \times$ TBST. Goat anti-rabbit IgG HRP (Abcam, ab6721) and goat anti-mouse IgG HRP antibodies (Abcam, ab6789) were used as secondary antibodies. Proteins were detected using ECL western blotting substrate (Promega, W1015).

\section{Immunocytochemistry (ICC) and immunohistochemistry (IHC)}

Cells were fixed in $95 \%$ ethanol and embedded in paraffin. Egg albumin was used for cell aggregation. For ICC and IHC, $4 \mu \mathrm{m}$ thick sections from FFPE tissue blocks were cut using a microtome and routinely deparaffinized. The sections were incubated with $0.3 \%$ hydrogen peroxide to block endogenous peroxidase activity. Antigen retrieval was performed in $0.01 \mathrm{M}$ of citrate buffer (pH 6.0) or Tris-EDTA buffer $(10 \mathrm{mM}$ Tris at $\mathrm{pH} 9.0$, $1 \mathrm{mM}$ EDTA, $0.03 \%$ Tween 20$)$ at $95{ }^{\circ} \mathrm{C}$. Three different PD-L1 antibodies (DAKO 22C3, 1:50; VENTANA SP142, 1:50; and VENTANA SP263, 1:50) were used for immunocytochemical and immunohistochemical staining. For SP142 IHC amplification and DAB development, the Biotin-Free Catalyzed Amplification System (DAKO, K1497) was used. For SP263 IHC amplification and DAB development, the OptiView DAB IHC Detection Kit (VENTANA, 760-700) was used according to the manufacturer's instructions. Each slide was counterstained with hematoxylin and then mounted.

To evaluate the IHC results of tissue samples including both whole sections and TMAs, each case was separated into groups with $<1 \%$ (negative), $1-49 \%$ (low), or $\geq 50 \%$ (high) positive tumor cells. A tumor cell with membranous staining, at least weak and partial, counted as a positive tumor cell. 


\section{Flow cytometry}

Cells were washed with fluorescence-activated cell sorting (FACS) buffer (filtered 0.1\% BSA in PBS) and stained with phycoerythrin (PE)-conjugated monoclonal antibody specific for PD-L1 (eBioscience, MIH1) or IgG (Miltenyi Biotec, 130-092-212). Cells were filtered using a Falcon $5 \mathrm{ml}$ round bottom tube with a cell strainer snap cap (Corning, 352235). Flow cytometric analysis was performed with FACSVerse and FACSuite (BD Biosciences).

\section{RNA extraction, CDNA synthesis, and quantitative reverse transcription PCR (qRT-PCR)}

Total RNA was isolated using RNeasy Mini Kit (Qiagen, 74106) according to the manufacturer's instructions and quantified using Nanodrop $^{\mathrm{TM}} 2000$ spectrophotometer (Thermo Fisher Scientific, ND-2000). One microgram of total RNA was used for the synthesis of cDNA. The cDNA was synthesized using the SuperScript III FirstStrand Synthesis System (Invitrogen). To determine mRNA levels of $P D-L 1$ and STAT1, qRT-PCR was performed using SYBR Green PCR Master Mix (Applied Biosystems, 4367659) and specific primer sets (Additional file 1: Table S3). Relative mRNA expression levels were normalized to the expression level of CTBP1 using $2^{-\Delta \Delta \mathrm{Ct}}$ (mean fold change).

\section{Statistical analysis}

Continuous variables were tested for normality of distribution using the Kolmogorov-Smirnov test and ShapiroWilk test. Unpaired t-test was used for the continuous variables fitting a normal distribution. Mann-Whitney U-test was used for the continuous variables showing a skewed distribution. Categorical variables were compared using the Chi square test or Fisher's exact test. RFS was defined as the time interval between initial resection and tumor recurrence or last follow-up. OS was defined as the time interval between the initial diagnosis and death or last follow-up. Survival analysis was performed using the Kaplan-Meier method with the log-rank test. P-values $\leq 0.05$ (2-tailed) was considered statistically significant. Statistical analyses were performed using Prism v.7 (GraphPad) and SPSS version 17.0 (SPSS Inc.).

\section{Results}

\section{Status of PD-L1 expression in various sarcoma cell lines}

To evaluate the expression levels of total PD-L1 protein, we performed western blot on 16 human sarcoma cell lines (Fig. 1A). PD-L1 expression levels were highly elevated in the HS-RMS-1, HT1080, and LP6 cell lines, while no detectable PD-L1 expression levels were observed in the A673, LIPO-246, MG-63, NMFH-1, and RH41 cell lines. Next, to measure the expression levels of PD-L1, which is present on the cell surface, FACS was performed using the same cell lines (Fig. 1B). Consistent with the results obtained from the western blot analysis, the HS-RMS-1, HT1080, and LP6 cell lines had higher PD-L1 expression levels. Additionally, increased PD-L1 expression was found in MLS402, MLS1765, and U2-OS cell lines.

Furthermore, we prepared FFPE cell blocks with the same cell lines and then performed ICC using anti-PDL1 antibodies (22C3 and SP142 clones) (Fig. 1C, D). To complement the fact that staining intensity of the SP142 clone has been known to be weak relative to that of the $22 \mathrm{C} 3$ clone, tyramide signal amplification was utilized for the IHC analysis $[15,16]$. Immunostaining using the 22C3 clone demonstrated PD-L1 expression only in the HS-RMS-1, HT1080, and LP6 cell lines, consistent with the results obtained from the western blot analysis. In contrast, with the SP142 clone, PD-L1 expression was detected in 10 human sarcoma cell lines including HSRMS-1, HT1080, and LP6.

Taken together, these data indicated that pleomorphic rhabdomyosarcoma, fibrosarcoma, and DDLPS PD-L1 expressed high level of PDL-1, as demonstrated by western blotting, FACS, and ICC results (Table 1).

\section{Status of PD-L1 expression in various sarcoma patient tissues}

Although pleomorphic rhabdomyosarcoma and fibrosarcoma cell lines showed PD-L1 immunoreactivity, these entities are rare. Therefore, we excluded them from the further evaluation using patient tissue. We included UPS and conventional osteosarcoma in consideration of their prevalence and the results of the previous studies. The results of the PD-L1 immunoreactivity evaluation are summarized in Table 2. In TMAs, no (0/28) DDLPS cases showed immunoreactivity with the PD-L1 (22C3) antibody, and one (1/29) DDLPS case showed immunoreactivity with PD-L1 (SP142) antibody in $\geq 1 \%$ of tumor cells. UPS showed $\geq 1 \%$ and $\geq 50 \%$ immunoreactivity in $20 \%(12 / 60)$ and $10 \%(6 / 60)$ of the cases, respectively, with the PD-L1 (22C3) antibody. With the PD-L1 (SP142) antibody, UPS showed $\geq 1 \%$ and $\geq 50 \%$ immunoreactivity in $16.3 \%$ (15/92) and 7.6\% (7/92) of the cases, respectively. With PD-L1 (SP263) antibody, UPS showed $\geq 1 \%$ and $\geq 50 \%$ immunoreactivity in $17.6 \%(9 / 51)$ and $9.8 \%$ $(5 / 51)$ of the cases, respectively. In whole sections stained with the PD-L1 (22C3) antibody, DDLPS showed $\geq 1 \%$ and $\geq 50 \%$ immunoreactivity in $21.9 \%(7 / 32)$ and $9.3 \%$ $(3 / 32)$ of the cases, respectively. Finally, osteosarcomas showed $\geq 50 \%$ immunoreactivity in $3.2 \%(1 / 31)$ of the cases. Representative images of PD-L1-positive and -negative staining for each histologic subtype for both TMAs and whole sections are shown in Fig. 2. In a comparison 


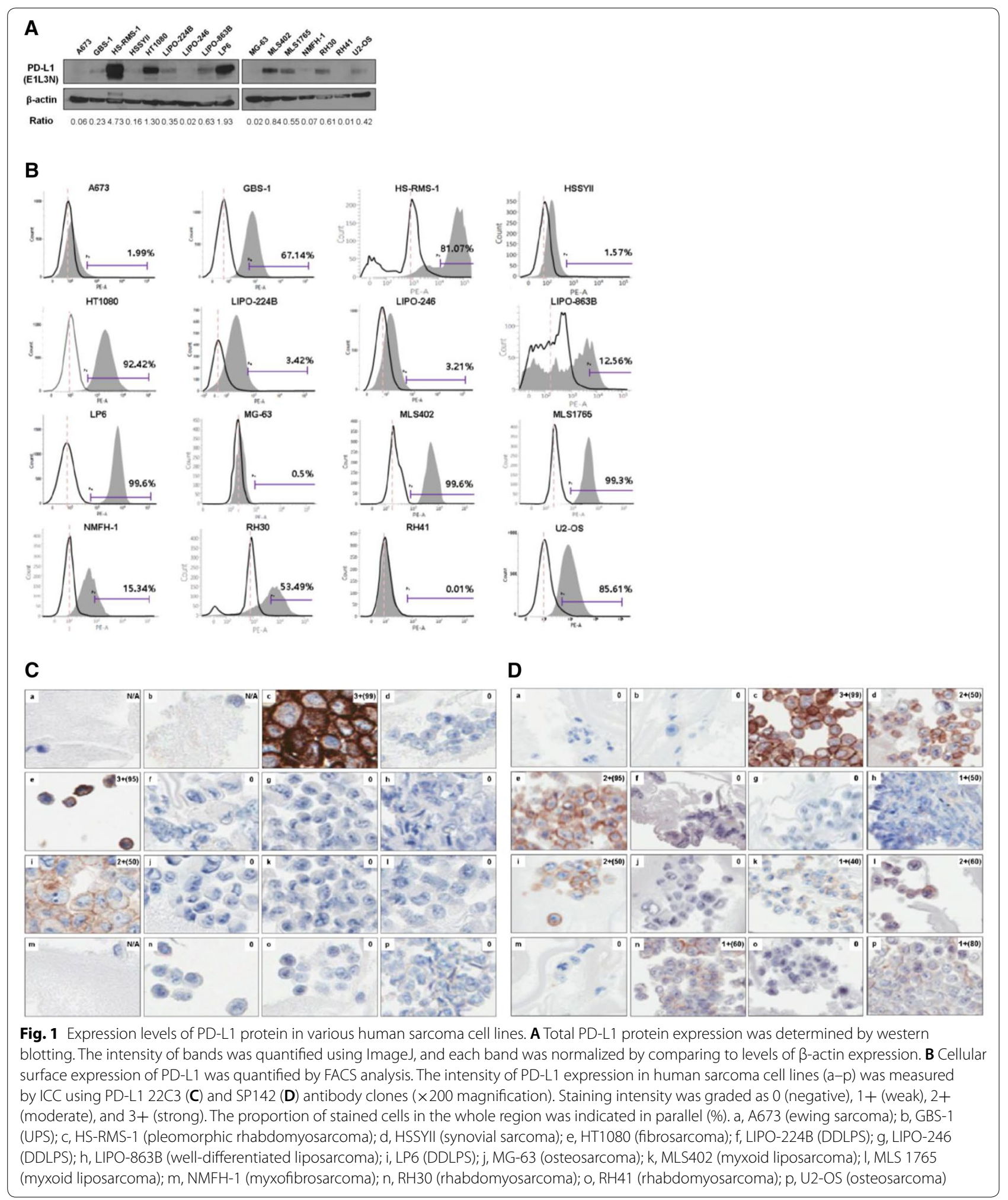


Table 1 Summary of the expression status of PD-L1 in human sarcoma cell lines

\begin{tabular}{|c|c|c|c|c|c|}
\hline Cell line & Origin & $\begin{array}{l}\text { WB (PD-L1/ } \beta \text {-actin } \\
\text { ratio) }\end{array}$ & FACS (\%) & ICC (22C3) & ICC (SP142) \\
\hline A673 & Ewing sarcoma & 0.06 & 1.99 & N/A & 0 \\
\hline GBS-1 & UPS & 0.23 & 67.14 & N/A & 0 \\
\hline HS-RMS-1 & Pleomorphic rhabdomyosarcoma & 4.73 & 81.07 & $3+$ & $3+$ \\
\hline HSSYII & Synovial sarcoma & 0.16 & 1.57 & 0 & $2+$ \\
\hline HT1080 & Fibrosarcoma & 1.30 & 92.42 & $3+$ & $2+$ \\
\hline LIPO-224B & DDLPS & 0.35 & 3.42 & 0 & $1+$ \\
\hline LIPO-246 & DDLPS & 0.02 & 3.21 & 0 & 0 \\
\hline LIPO-863B & WDLPS & 0.63 & 12.56 & 0 & $1+$ \\
\hline LP6 & DDLPS & 1.93 & 99.6 & $2+$ & $2+$ \\
\hline MG-63 & Osteosarcoma & 0.02 & 0.5 & 0 & 0 \\
\hline MLS402 & Myxoid liposarcoma & 0.84 & 99.6 & 0 & $1+$ \\
\hline MLS1765 & Myxoid liposarcoma & 0.55 & 99.3 & 0 & $2+$ \\
\hline $\mathrm{NMFH}-1$ & Myxofibrosarcoma & 0.07 & 15.34 & $\mathrm{~N} / \mathrm{A}$ & 0 \\
\hline $\mathrm{RH} 30$ & Rhabdomyosarcoma & 0.61 & 53.49 & 0 & $1+$ \\
\hline $\mathrm{RH} 41$ & Rhabdomyosarcoma & 0.01 & 0.01 & 0 & 0 \\
\hline U2-OS & Osteosarcoma & 0.42 & 85.61 & 0 & $1+$ \\
\hline
\end{tabular}

The staining intensity was graded as 0 (negative), $1+$ (weak), $2+$ (moderate), and $3+$ (strong)

UPS undifferentiated pleomorphic sarcoma, DDLPS dedifferentiated liposarcoma, WDLPS well-differentiated liposarcoma, N/A not available, WB western blot, FACS fluorescence-activated cell sorting, ICC immunocytochemistry

of the three PD-L1 antibodies, 22C3 and SP263 showed a strong correlation (Pearson's $r=0.882$ ), but SP142 showed only moderate correlation with 22C3 (Pearson's $r=0.551$ ) and SP263 (Pearson's $r=0.503$ ) (Additional file 1: Table S4).

\section{Association between PD-L1 expression and clinicopathological features in DDLPS or UPS}

The association between the PD-L1 expression and the clinicopathologic parameters are only available in the DDLPS whole section group $(\mathrm{N}=32)$ and UPS TMA group $(\mathrm{N}=60)$. The remaining groups could not be evaluated due to too their low positive rate.

The clinicopathologic features of the DDLPS whole section group and UPS TMA group are summarized in Table 3. In the DDLPS group, after a median follow-up duration of 19 months, 11 patients (34.4\%) had experienced recurrence, and three patients (9.4\%) had died at the time of survival analysis. There were no statistically significant differences between the no PD-L1 and $\geq 1 \%$ PD-L1 expression group for clinicopathological parameters such as age, sex, tumor size, FNCLCC grade, resection margin status, and history of chemotherapy and radiation therapy. However, the RFS and OS rates were statistically significantly different between the patients with DDLPS with no PD-L1 expression and those with $\geq 1 \%$ PD-L1 expression (Fig. 3). In the UPS group, after a median follow-up duration of 49 months, 27 patients (45.0\%) had experienced recurrence, and 36 patients
(60.0\%) had died at the time of the survival analysis. There were no statistically significant differences between the PD-L1 no expression group and $\geq 1 \%$ PD-L1 expression group for the clinicopathological parameters including RFS and OS (Fig. 3).

Induction of PD-L1 by IFN- $\gamma$ treatment in sarcoma cell lines Several recent studies have reported that IFN- $\gamma$ induces PD-L1 expression in tumor cells by activating STAT1 signaling, suggesting a possibility on the effectiveness of immunotherapy targeting IFN- $\gamma$-induced PD-L1 expression [17-20]. Based on these findings, we examined whether IFN- $\gamma$ treatment induces PD-L1 expression in sarcoma cell lines with IFN- $\gamma$. We treated the 16 sarcoma cell lines with IFN- $\gamma$, then measured the mRNA expression levels of STAT1 and PD-L1 using qRT-PCR assay.

Upon IFN- $\gamma$ treatment, the mRNA expression levels of both STAT1 and PD-L1 increased in the HS-RMS-1, LIPO-224B, MLS1765, RH30, and RH41 cell lines (Fig. 4A, B). These results suggest that $P D-L 1$ expression was induced by IFN- $\gamma$, and raises the possibility of dual therapy using IFN- $\gamma$ and PD-L1 for pleomorphic rhabdomyosarcoma, DDLPS, myxoid liposarcoma, and rhabdomyosarcoma.

\section{Discussion}

Recent advances from molecular analyses have revealed that many sarcomas are not only morphologically but also genetically distinct neoplasms. According to 


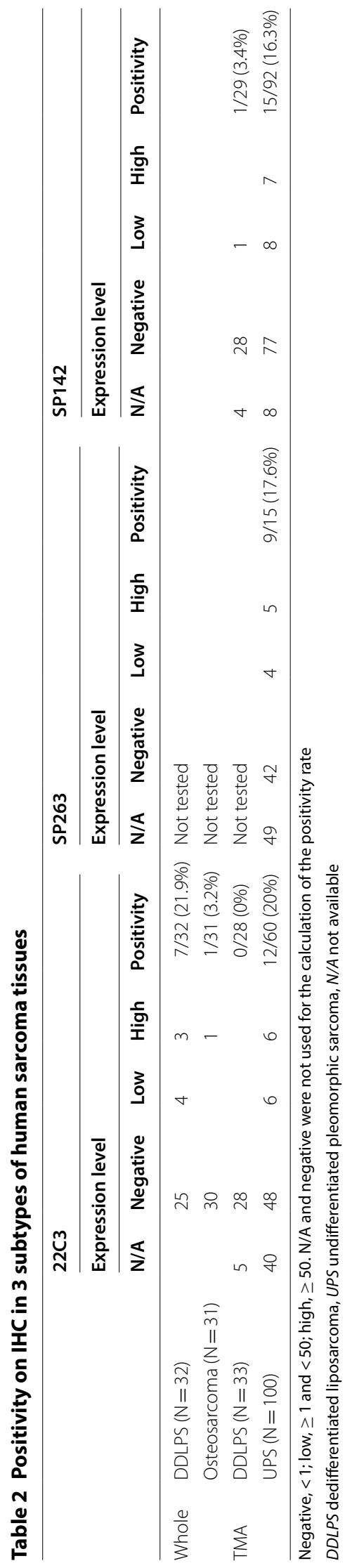




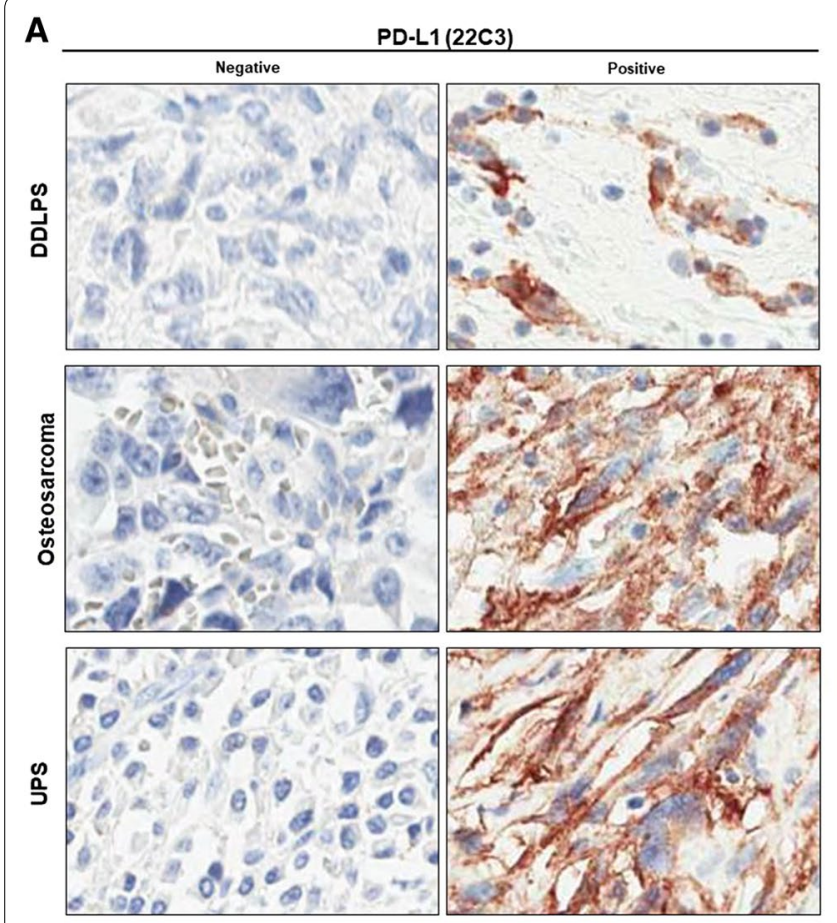

B
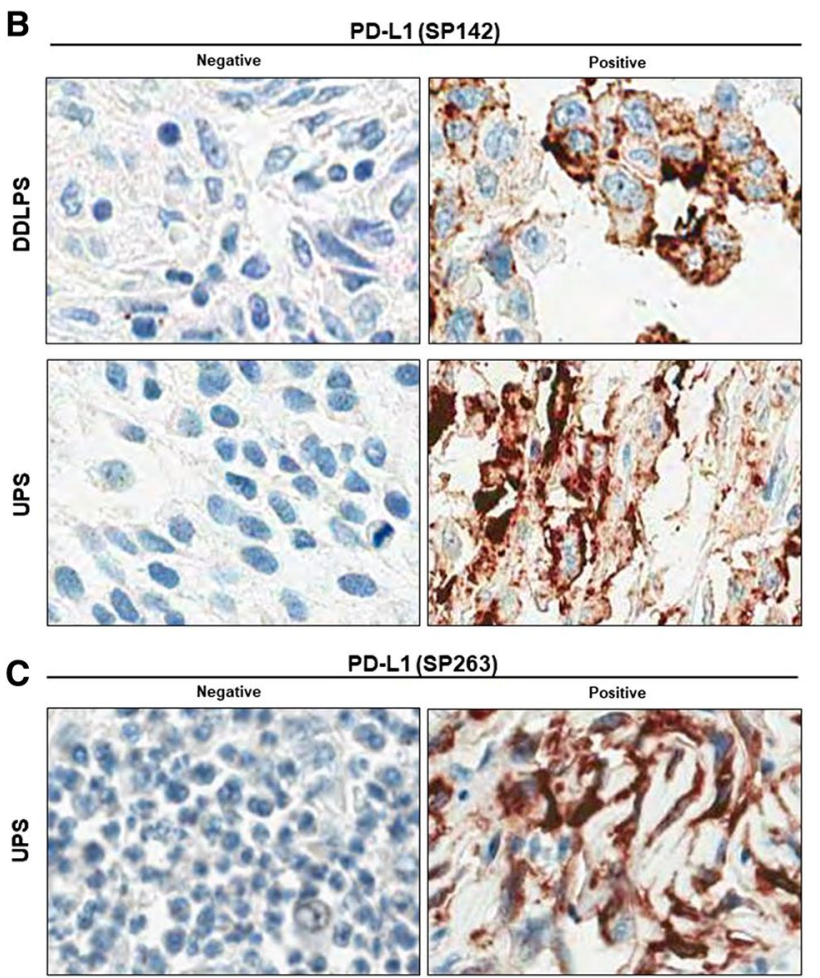

Fig. 2 Representative cases of PD-L1 IHC in 3 sarcoma subtype tissues. Intensity of PD-L1 expression in DDLPS, osteosarcoma, and UPS was measured by IHC using PD-L1 22C3 (A), SP142 (B), and SP263 (C) antibody clones (×200 magnification)

Table 3 Clinicopathologic characteristics of DDLPS (whole section) and UPS (TMA)

\begin{tabular}{|c|c|c|c|c|c|c|c|c|}
\hline & DDLPS, whole & section & & & UPS, TMA & & & \\
\hline & Total & Negative & Positive & $P$ & Total & Negative & Positive & $P$ \\
\hline N & 32 & 25 & 7 & & 60 & 48 & 12 & \\
\hline Age, year (median) & 56 & 55 & 58 & 0.616 & 53 & 55 & 42.5 & 0.132 \\
\hline$M: F$ & 19:13 & $15: 10$ & $4: 3$ & $>0.99$ & $32: 28$ & $28: 20$ & $4: 8$ & 0.121 \\
\hline Chemotherapy, n (\%) & $6 / 32(18.8 \%)$ & $5 / 25(20.0 \%)$ & $1 / 7(14.3 \%)$ & $>0.99$ & $27 / 60(45.0 \%)$ & $21 / 48(43.8 \%)$ & $6 / 12(50 \%)$ & 0.754 \\
\hline Radiation therapy, n (\%) & $20 / 32(62.5 \%)$ & $15 / 25(60.0 \%)$ & $5 / 7(71.4 \%)$ & 0.683 & $30 / 60(50 \%)$ & $22 / 48(45.8 \%)$ & $8 / 12(66.7 \%)$ & 0.197 \\
\hline Recurrence, n (\%) & $11 / 32(34.4 \%)$ & $8 / 25(32.0 \%)$ & $3 / 7(42.9 \%)$ & & $27 / 60(45.0 \%)$ & $22 / 48(45.8 \%)$ & $5 / 12(41.7 \%)$ & \\
\hline Expire, n (\%) & $3 / 32(9.4 \%)$ & $1 / 25(4.0 \%)$ & $2 / 7(28.6 \%)$ & & $36 / 60(60.0 \%)$ & 29/48 (60.4\%) & $7 / 12(58.3 \%)$ & \\
\hline Tumor size (cm, median) & 12.75 & 12.5 & 14 & 0.569 & 6 & 5.35 & 7 & 0.523 \\
\hline Resection margin involved, $\mathrm{n}(\%)$ & $28 / 32(87.5 \%)$ & $22 / 25(88.0 \%)$ & $6 / 7(85.7 \%)$ & $>0.99$ & $25 / 49(51.0 \%)$ & 21/39 (53.8\%) & $4 / 10(40.0 \%)$ & 0.496 \\
\hline FNCLCC & & & & & & & & \\
\hline 2 & 28/32 (87.5\%) & 23/25 (92.0\%) & $5 / 7(71.4 \%)$ & 0.201 & $17 / 41(41.5 \%)$ & 14/33 (42.4\%) & $3 / 8(37.5 \%)$ & $>0.99$ \\
\hline 3 & $4 / 32(12.5 \%)$ & $2 / 25(8.0 \%)$ & $2 / 7(28.6 \%)$ & & $24 / 41(58.5 \%)$ & 19/33 (57.6\%) & $5 / 8(62.5 \%)$ & \\
\hline
\end{tabular}

Negative, no PD-L1 immunoreactivity; positive, $\geq 1 \%$ PD-L1 immunoreactivity

DDLPS dedifferentiated liposarcoma, UPS undifferentiated liposarcoma, RFS recurrence free survival

genomic profiles, soft tissue tumors can be broadly divided into two groups $[4,21]$. The first group comprises tumors associated with specific genetic alterations and relatively simple karyotypes. Sarcoma subtypes belonging to this group include translocation-associated tumors, such as synovial sarcoma and myxoid liposarcoma, and mutation-associated tumors such as a gastrointestinal stromal tumor. Because there is a specific genomic alteration in each subtype, gene-targeted therapies are currently under investigation. On the other hand, the second 

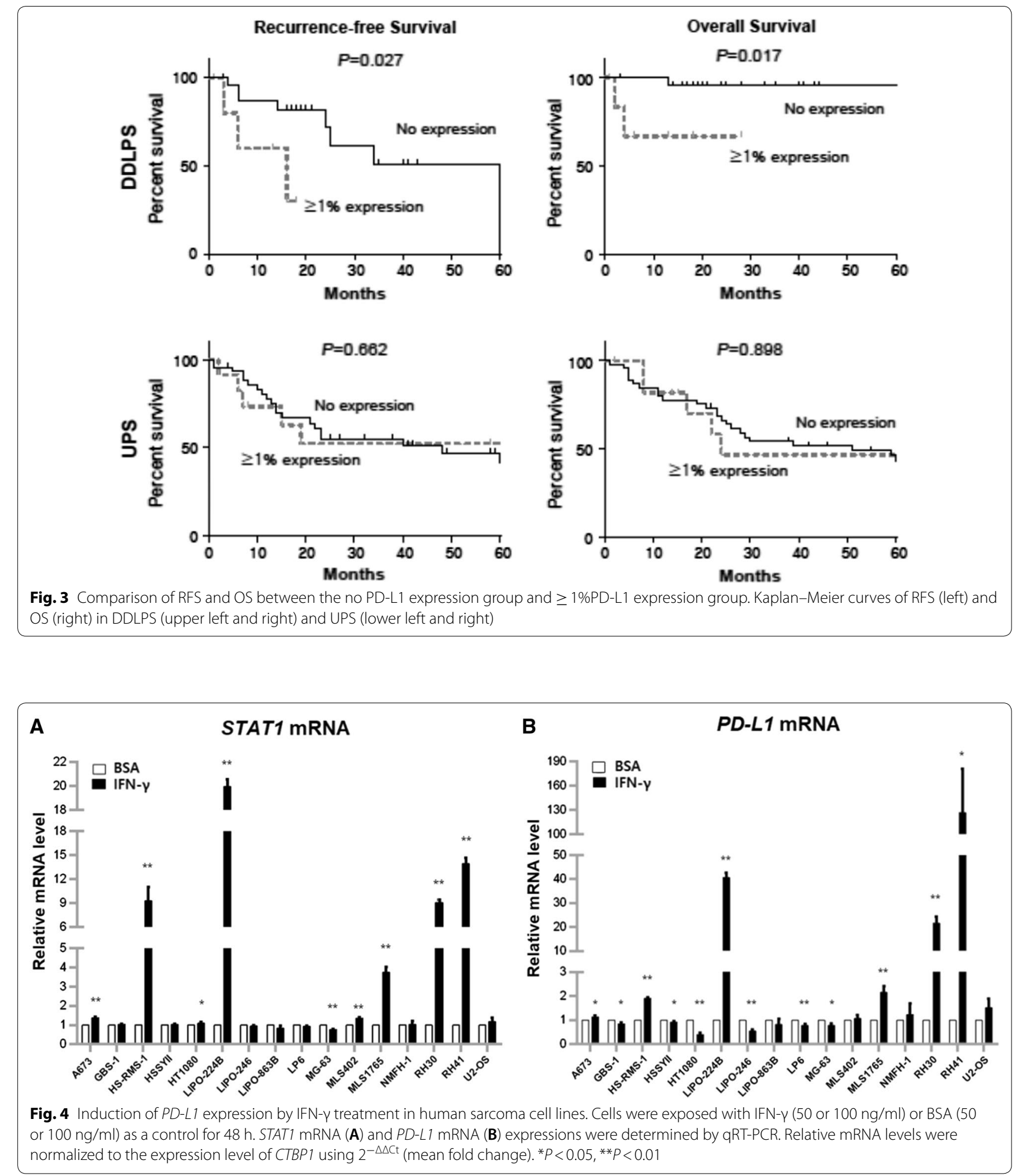

group of sarcomas is those with complex karyotypes [3]. Sarcoma subtypes belong to this group includes UPS, DDLPS, pleomorphic rhabdomyosarcoma, myxofibrosarcoma, and malignant peripheral nerve sheath tumor
$[21,22]$. Because most of these entities have no diseasespecific genomic alterations, gene-targeted therapies are of limited value. However, several recent studies have revealed that the tumors with complex karyotypes emit 
signals that increase their immunogenicity, and evasion of local immune surveillance plays an important role in their tumorigenesis [23]. Although the mechanisms of immune evasion are still unknown, in our opinion, the immune evasion via PD-L1 expression could be an important component.

In this study, we firstly examined the prevalence of PD-L1 expression in in a 16 sarcoma cell lines comprising 12 different subtypes. Pleomorphic rhabdomyosarcoma (HS-RMS-1), fibrosarcoma (HT1080), and DDLPS (LP6) cell lines showed a consistently increased PD-L1 protein expression via western blot, FACS, and ICC by $22 \mathrm{C} 3$ antibody. In a review of their genetic characteristics, both pleomorphic rhabdomyosarcoma and fibrosarcoma have been known to have complex karyotypes without specific genomic alterations [22, 24]. In cases of DDLPS, although it is defined by 12q13 15 amplifications, it is currently classified as a complex karyotype group because of additional genomic alterations during dedifferentiation [21]. In summary, cell lines that showed high PD-L1 expression all belonged to the complex karyotype group, which was consistent with our theory and a previous report [25]. On the other hand, UPS (GBS-1) and myxofibrosarcoma (NMFH-1) cell lines did not show PD-L1 expression despite their complex karyotype [21]. However, our IHC results revealed a $20 \%(12 / 60)$ expression rate in the TMAs of UPS. Therefore, we suggest that high PD-L1 expression tend to match with a complex karyotype group, although not all tumors with a complex karyotype express PD-L1. Screening methods such as IHC would be required in practice for the patient selection, which is similar for non-small cell carcinoma.

Although there have been several previous studies that focused on the PD-L1 immunohistochemical expression in sarcomas, most previous studies are based on a small number of specimens and showed controversial results. For example, the PD-L1 expression rates of leiomyosarcoma have been reported as $0 \%(0 / 4), 11 \%(1 / 9)$, and $70 \%$ $(14 / 20)[11,13,26]$. In this study, we used 230 sarcoma tissue samples, comprised of 87 whole sections and 143 TMAs, and three different anti-PD-L1 antibodies to solve this controversy. Our overall expression rate was $10.9 \%$ (20/184), which was lower than that of several previous studies $(43-58 \%)$ [11, 13]. However, several other studies have also reported overall expression rates similar to our study $(5-12 \%)[14,27]$. In comparison with our study and previous studies, we suggest that the overall expression rates could be related to the anti-PD-L1 antibody used for IHC. More recent studies and our study used the 22C3 clone of anti-PD-L1 antibody, which is currently used in practice, and showed similar overall expression rates. It is a well-known problem that the expression rates of PD-L1 could vary according to the antibodies used in the IHC.
The Blueprint Project, which included four anti-PD-L1 antibodies used in clinical trials, showed that the staining proportion of tumor cells could vary according to the antibody clone that was used [28]. These discordances were most significant with the SP142 clone compared to the 22C3, 28-8, and SP263 clones [28]. Our results showed a strong correlation (Pearson's $r=0.882$ ) between 22C3 and SP263, but SP142 showed only moderate correlation with 22C3 (Pearson's $r=0.551$ ) and SP263 (Pearson's $r=0.503$ ), which was consistent with the Blueprint Project.

Histologic characteristics also can increase interobserver variability in the evaluation of $\mathrm{PD}-\mathrm{L} 1$ expression of sarcomas. Under a light microscope, many sarcomas, especially UPS and DDLPS, show tumor cells intermixed with inflammatory cells. According to a recent molecular analysis report, UPS and DDLPS showed the highest median number of macrophages among sarcomas [21]. Considering that the inflammatory cells including macrophages can show PD-L1 immunoreactivity, the interpretation of PD-L1 immunoreactivity in sarcomas is not straightforward and can result in high interobserver variability. Additionally, as we described above, the recent molecular analysis revealed that many histologic sarcoma subtypes have distinct molecular characteristics. Considering that different sarcoma subtype shows different morphologies and genetics, we hypothesize that different sarcoma subtypes would show different overall PD-L1 expression rates.

DDLPS showed no PD-L1 expression in the TMAs but showed a $21.9 \%(7 / 32)$ PD-L1 expression rate in whole sections. This result may have been mainly due to low tumor cell proportion with PD-L1 positivity. In this study, only 3 cases showed PD-L1 expression in more than $50 \%$ of tumor cells and are usually limited to a dedifferentiated area. In a review of previous studies, there were no positive cases in the SARC028 study, which was based on biopsy samples [14]. Torabi et al. [29] reported that only one weak positive case in 64 liposarcoma cases, but they did not include DDLPS cases in their study. Osteosarcomas had a PD-L1 expression rate of 3.2\% (1/31). The only case with PD-L1 immunoreactivity was conventional osteosarcoma with high-grade spindle cell morphology. Similar to our results, Torabi et al. [29] reported no $(0 / 26)$ PD-L1 expression in osteosarcomas. There was also a previous study that reported that $24 \%(9 / 38)$ cases of osteosarcoma showed high PD-L1 RNA expression, but they did not report the immunoreactivity of PD-L1 protein in their study [30]. UPS had a 20\% (12/60) PD-L1 expression rate in our study. This result was consistent with the SARC028 study which reported that only UPS cases (3/10) showed PD-L1 immunoreactivity [14]. However, both the SARC028 study and our study are based 
on biopsied specimens. Considering the difference in expression rates between TMAs and whole sections of DDLPS in our study, there is a possibility of underestimating the overall expression rate in UPS. In summary, in this study, we confirmed positive PD-L1 expression with the 22C3 clone in DDLPS and UPS. Our finding supports a result where pembrolizumab have a specific activity in patients with DDLPS and UPS among seven subtypes of sarcomas, which was comprised of 84 patients with bone or soft tissue sarcomas in the SARC028 study [14].

The association between PD-L1 expression and poor prognosis had been reported in many previous studies $[13,25,30]$. The cases of DDLPS in our study also showed significantly worse outcomes, whereas cases of UPS in our study showed no significant differences in outcome. Considering that UPS is a sarcoma with very poor prognosis, the contribution of PD-L1 expression to prognosis could be masked by the aggressive of the UPS.

IFN- $\gamma$ has been reported to induce PD-L1 expression in several cell lines including chordoma, angiosarcoma, and osteosarcoma cell lines [17, 19, 30]. Noteworthy, PD-L1 expression was induced following vascular-targeted photodynamic treatment or ionizing radiotherapy through an increase in IFN- $\gamma$, then a mono- or combination treatment with systemic PD-1/PD-L1 pathway blockade inhibits the generation of potent local and systemic tumors in mouse models using human renal cells or murine colon cancer cells [31, 32]. These previous reports suggest the possible effectiveness of antitumor immunotherapy through a dual treatment with IFN- $\gamma$ and PD-L1. We also observed the possibility of dual therapy using IFN- $\gamma$ and PD-L1 in pleomorphic rhabdomyosarcoma, DDLPS, myxoid liposarcoma, and rhabdomyosarcoma. However, the effectiveness of dual therapy with IFN- $\gamma$ and PD-L1 will need to be validated in various sarcoma subtypes in vivo.

\section{Conclusions}

The encouraging result from the SARC028 study revealed the possibility of anti-PD-L1 therapy for sarcoma treatments. Precise patient selection would be mandatory for further evaluations. We showed that PD-L1 immunohistochemical reactivity could be influenced by histologic subtypes, tissue acquisition methods, PD-L1 primary antibody clone, and difficulties in interpretation. Nevertheless, anti-PD-L1 therapy in sarcomas is still largely unexplored, and further studies would allow for better patient selection and new therapeutic strategies.

\section{Additional files}

Additional file 1: Table S1. Subtype and source of soft tissue cell lines. Table S2. Cell culture medium. Table S3. Primer sets of qRT-PCR. Table S4. Correlation of IHC with 3 anti-PD-L1 clones in UPS tissues (A) $N=46$ (without N/A), correlation coefficient. (B) $N=100$ (with N/A), correlation coefficient. (C) $\mathrm{N}=13$ (with positive expression cases in A), correlation coefficient.

\section{Abbreviations}

PD-L1: programmed death-ligand 1; DDLPS: dedifferentiated liposarcoma; TMA: tissue microarray; UPS: undifferentiated pleomorphic sarcoma; RFS: recurrence-free survival; OS: overall survival; IFN-ү: interferon-gamma; PD-1: programmed cell death protein 1; FFPE: formalin-fixed paraffin-embedded; ATCC: American Type Culture Collection; KCLB: Korean Cell Line Bank; ICC: immunocytochemistry; IHC: immunohistochemistry; FACS: fluorescence activated cell sorting; PE: phycoerythrin.

\section{Authors' contributions}

HKP, YJK, and YLC designed the study. HKP, MGK, MJS, and YJK wrote the manuscript. HKP and YLC reviewed the histopathology and IHC results. HKP, MGK, MJS, SEL, and YJK performed the clinical analysis and experiments. HKP, YJK, and YLC provided critical comments on the manuscript. All authors read and approved the final manuscript.

\section{Author details \\ 1 Department of Pathology, Konkuk University Medical Center, Konkuk University School of Medicine, Seoul, South Korea. ${ }^{2}$ Department of Health Sci- ences and Technology, SAIHST, Sungkyunkwan University, Seoul, South Korea. \\ ${ }^{3}$ Laboratory of Cancer Genomics and Molecular Pathology, Samsung Medical Center, Sungkyunkwan University School of Medicine, Irwon-ro 81, Gang- nam-gu, Seoul 06351, South Korea. ${ }^{4}$ Department of Pathology and Transla- tional Genomics, Samsung Medical Center, Sungkyunkwan University School of Medicine, Irwon-ro 81, Gangnam-gu, Seoul 06351, South Korea.}

\section{Acknowledgements}

We would like to thank Dr. Hiroaki Kanda, Dr. Hiroshi Sonobe, Dr. Dina Lev, Dr. Jonathan A. Fletcher, Dr. Pierre Åman, Dr. Akira Ogose and Dr. Peter J. Houghton for their generous gifts and Dr. Ka-Won Noh (SAIHST, Sungkyunkwan University) for technical supports.

\section{Competing interests}

The authors declare that they have no competing interests.

Availability of data and materials

Please contact author for data requests.

Consent for publication

Not applicable.

\section{Ethics approval and consent to participate}

This study was approved by the Institutional Review Board of Samsung Medical Center in Seoul, Korea (IRB File No. 2018-03-143).

Funding

This work was supported by a National Research Foundation of Korea (NRF) Grant funded by the Korean Government (Grant Number 2013R1A1A2011536 and 2016R1A5A2945889).

\section{Publisher's Note}

Springer Nature remains neutral with regard to jurisdictional claims in published maps and institutional affiliations. 
Received: 27 May 2018 Accepted: 9 October 2018

Published online: 06 November 2018

\section{References}

1. Surveillance, Epidermiology, and End Results (SEER) Program (www.seer. cancer.gov) SEER*Stat Database: incidence-SEER 9 regs reseach data, Nov 2010 sub (1973-2008). 1969-2009 Counties, National Cancer Institute, DCCPS; 2011.

2. Burningham Z, Hashibe M, Spector L, Schiffman JD. The epidemiology of sarcoma. Clin Sarcoma Res. 2012;2:14.

3. Linch M, Miah AB, Thway K, Judson IR, Benson C. Systemic treatment of soft-tissue sarcoma-gold standard and novel therapies. Nat Rev Clin Oncol. 2014;11:187-202

4. Frezza AM, Stacchiotti S, Gronchi A. Systemic treatment in advanced soft tissue sarcoma: what is standard, what is new. BMC Med. 2017;15:109.

5. Santoro A, Tursz T, Mouridsen H, Verweij J, Steward W, Somers R, Buesa J, Casali P, Spooner D, Rankin E, et al. Doxorubicin versus CYVADIC versus doxorubicin plus ifosfamide in first-line treatment of advanced soft tissue sarcomas: a randomized study of the European organization for research and treatment of cancer soft tissue and bone sarcoma group. J Clin Oncol. 1995;13:1537-45.

6. Ishida Y, Agata Y, Shibahara K, Honjo T. Induced expression of PD-1, a novel member of the immunoglobulin gene superfamily, upon programmed cell death. EMBO J. 1992;11:3887-95.

7. Rosenblatt J, Avigan D. Targeting the PD-1/PD-L1 axis in multiple myeloma: a dream or a reality? Blood. 2017;129:275-9.

8. Iwai Y, Hamanishi J, Chamoto K, Honjo T. Cancer immunotherapies targeting the PD-1 signaling pathway. J Biomed Sci. 2017;24:26.

9. Cho JH, Zhou W, Choi YL, Sun JM, Choi H, Kim TE, Dolled-Filhart M, Emancipator K, Rutkowski MA, Kim J. Retrospective molecular epidemiology study of PD-L1 expression in patients with EGFR-mutant non-small cell lung cancer. Cancer Res Treat. 2018;50:95-102.

10. Lee HS, Kim WH, Kwak Y, Koh J, Bae JM, Kim KM, Chang MS, Han HS, Kim JM, Kim HW, et al. Molecular testing for gastrointestinal cancer. J Pathol TransI Med. 2017;51:103-21.

11. Kim JR, Moon YJ, Kwon KS, Bae JS, Wagle S, Kim KM, Park HS, Lee H, Moon WS, Chung MJ, et al. Tumor infiltrating PD1-positive lymphocytes and the expression of PD-L1 predict poor prognosis of soft tissue sarcomas. PLoS ONE. 2013;8:1-9.

12. Movva S, Wen W, Chen W, Millis SZ, Gatalica Z, Reddy S, von Mehren M, Van Tine BA. Multi-platform profiling of over 2000 sarcomas: identification of biomarkers and novel therapeutic targets. Oncotarget. 2015;6:12234-47.

13. Kim C, Kim EK, Jung H, Chon HJ, Han JW, Shin K-H, Hu H, Kim KS, Choi YD, Kim S, et al. Prognostic implications of PD-L1 expression in patients with soft tissue sarcoma. BMC Cancer. 2016;16:434.

14. Tawbi HA, Burgess M, Bolejack V, Van Tine BA, Schuetze SM, Hu J, D'Angelo $S$, Attia S, Riedel RF, Priebat DA, et al. Pembrolizumab in advanced softtissue sarcoma and bone sarcoma (SARC028): a multicentre, two-cohort, single-arm, open-label, phase 2 trial. Lancet Oncol. 2017;18:1493-501.

15. Buttner R, Gosney JR, Skov BG, Adam J, Motoi N, Bloom KJ, Dietel M, Longshore JW, Lopez-Rios F, Penault-Llorca F, et al. Programmed deathligand 1 immunohistochemistry testing: a review of analytical assays and clinical implementation in non-small-cell lung cancer. J Clin Oncol. 2017:35:3867-76.

16. Rimm DL, Han G, Taube JM, Yi ES, Bridge JA, Flieder DB, Homer R, West WW, Wu H, Roden AC, et al. A prospective, multi-institutional, pathologist-based assessment of 4 immunohistochemistry assays for PD-L1 expression in non-small cell lung cancer. JAMA Oncol. 2017:3:1051-8.

17. Feng Y, Shen J, Gao Y, Liao Y, Cote G, Choy E, Chebib I, Mankin H, Hornicek F, Duan Z. Expression of programmed cell death ligand 1 (PD-L1) and prevalence of tumor-infiltrating lymphocytes (TILs) in chordoma. Oncotarget. 2015;6:11139-49.

18. Mandai M, Hamanishi J, Abiko K, Matsumura N, Baba T, Konishi I. Dual faces of IFNgamma in cancer progression: a role of PD-L1 induction in the determination of pro- and antitumor immunity. Clin Cancer Res. 2016;22:2329-34.

19. Honda Y, Otsuka A, Ono S, Yamamoto Y, Seidel JA, Morita S, Hirata M, Kataoka TR, Takenouchi T, Fujii K, et al. Infiltration of PD-1-positive cells in combination with tumor site PD-L1 expression is a positive prognostic factor in cutaneous angiosarcoma. Oncoimmunology. 2017;6:e1253657.

20. Marabelle A, Aspeslagh S, Postel-Vinay S, Soria JC. JAK mutations as escape mechanisms to Anti-PD-1 therapy. Cancer Discov. 2017;7:128-30.

21. Cancer Genome Atlas Research Network. Comprehensive and integrated genomic characterization of adult soft tissue sarcomas. Cell. 2017;171(950-965):e928.

22. Li G, Ogose A, Kawashima H, Umezu H, Hotta T, Tohyama T, Ariizumi T, Endo N. Cytogenetic and real-time quantitative reverse-transcriptase polymerase chain reaction analyses in pleomorphic rhabdomyosarcoma. Cancer Genet Cytogenet. 2009;192:1-9.

23. López-Soto A, Gonzalez S, López-Larrea C, Kroemer G. Immunosurveillance of malignant cells with complex karyotypes. Trends Cell Biol. 2017:27:880-4.

24. Dal Cin P, Pauwels P, Sciot R, Van den Berghe H. Multiple chromosome rearrangements in a fibrosarcoma. Cancer Genet Cytogenet. 1996;87:176-8.

25. Bertucci F, Finetti P, Perrot D, Leroux A, Collin F, Le Cesne A, Coindre JM, Blay JY, Birnbaum D, Mamessier E. PDL1 expression is a poor-prognosis factor in soft-tissue sarcomas. Oncolmmunology. 2017;6:1-11.

26. Paydas S, Bagir EK, Deveci MA, Gonlusen G. Clinical and prognostic significance of PD-1 and PD-L1 expression in sarcomas. Med Oncol. 2016:33:1-10

27. D'Angelo SP, Shoushtari AN, Agaram NP, Kuk D, Qin LX, Carvajal RD, Dickson MA, Gounder M, Keohan ML, Schwartz GK, Tap WD. Prevalence of tumor-infiltrating lymphocytes and PD-L1 expression in the soft tissue sarcoma microenvironment. Hum Pathol. 2015:46:357-65.

28. Hirsch FR, McElhinny A, Stanforth D, Ranger-Moore J, Jansson M, Kulangara K, Richardson W, Towne P, Hanks D, Vennapusa B, et al. PD-L1 immunohistochemistry assays for lung cancer: results from phase 1 of the blueprint PD-L1 IHC assay comparison project. J Thorac Oncol. 2017;12:208-22.

29. Torabi A, Amaya CN, Wians FH, Bryan BA. PD-1 and PD-L1 expression in bone and soft tissue sarcomas. Pathology. 2017:49:506-13.

30. Shen JK, Cote GM, Choy E, Yang P, Harmon D, Schwab J, Nielsen GP, Chebib I, Ferrone S, Wang $X$, et al. Programmed cell death ligand 1 expression in osteosarcoma. Cancer Immunol Res. 2014;2:690-8.

31. Dovedi SJ, Adlard AL, Lipowska-Bhalla G, McKenna C, Jones S, Cheadle EJ, Stratford IJ, Poon E, Morrow M, Stewart R, et al. Acquired resistance to fractionated radiotherapy can be overcome by concurrent PD-L1 blockade. Cancer Res. 2014;74:5458-68.

32. O'Shaughnessy MJ, Murray KS, La Rosa SP, Budhu S, Merghoub T, Somma A, Monette S, Kim K, Corradi RB, Scherz A, Coleman JA. Systemic antitumor immunity by PD-1/PD-L1 inhibition is potentiated by vasculartargeted photodynamic therapy of primary tumors. Clin Cancer Res. 2018;24:592-9. 